\title{
Treatment and management of patients with cardiovascular disease in General Practice;
}

\section{Underdiagnosed and undertreated}

Mülverstedt, S. ${ }^{1}$; Hildebrandt, P., M.D., DMSc²; Prescott, E., M.D., DMSc ${ }^{1}$; Heitmann, M., M.D., PhD ${ }^{1}$

${ }^{1}$ Dept. of Cardiology, Bispebjerg University Hospital, ${ }^{2}$ Heart Clinic Frederiksberg;

\section{BACKGROUND \& AIMS}

The Danish Heart Association estimates that approximately $10 \%$ of the Danish adult population have experienced one or more cardiovascular events. According to ESC Prevention Guidelines, LDL should be reduced to a level of $<1.8 \mathrm{mmol} / \mathrm{L}(70 \mathrm{mg} / \mathrm{dl})$ or at least $50 \%$ from baseline. In Denmark, following a cardiovascular event, patients are offered a rehabilitation programme and then discharged to follow up by their regular general practitioner (GP). In this study we examined the extent of lipid lowering therapy (LLT) and if the patients reach their LDL target level.

\section{METHOD}

By screening the patient records of individuals aged $18-100$ years at six GP clinics in Copenhagen we identified a CVD population with one or more measurements of LDL. In this population we registered information about LDL levels (maximum and the latest follow up), LLT and diagnoses of ischaemic heart disease, stroke and/or peripheral artery disease.

\section{RESULTS}

A total of 9,651 individuals were evaluated. In the population 278 individuals suffered CVD, corresponding to $2.88 \%$ ( $\mathrm{Cl}$ [2.54;3.21]). Of these patients 229 had one or more LDL measurements. Even though a significant reduction of LDL was observed in both patients with or without active LLT, only $26.2 \%$ (CI [20.5-31.9]) reached their LDL-C target.

\section{CONCLUSION}

It appears that a large proportion of CVD patients in general practice are not registered properly, and those who are registered do not meet their treatment target in respects to LDL. Furthermore, less than a third of the estimated CVD patients were registered.

\begin{tabular}{|c|c|c|c|}
\hline \multirow[t]{2}{*}{ TABLE 1} & \multicolumn{3}{|r|}{ BASELINE } \\
\hline & TOTAL & MEN & WOMEN \\
\hline $\mathrm{N}$ & 229 & 129 & 100 \\
\hline Age [yrs.] (Range) & $\begin{array}{r}67.3(25.0- \\
94.0)\end{array}$ & $\begin{array}{r}67.6(37.9- \\
92.5)\end{array}$ & $\begin{array}{r}66.9(25.0- \\
94.0)\end{array}$ \\
\hline IHD [n] (\%) & 110 (48.0\%) & 74 (67.3\%) & 36 (32.7\%) \\
\hline CVASC [n] (\%) & $102(44.5 \%)$ & $51(50.0 \%)$ & $51(50.0 \%)$ \\
\hline PAD [n] (\%) & 45 (19.7\%) & $23(51.1 \%)$ & $22(48.9 \%)$ \\
\hline $\begin{array}{l}\text { Max LDL-C [mmol/L] } \\
\text { (SD) }\end{array}$ & $3.80(1.207)$ & $3.78(1.244)$ & $3.83(1.163)$ \\
\hline $\begin{array}{l}\text { Latest LDL-C [mmol/L] } \\
\text { (SD) }\end{array}$ & $2.61(0.930)$ & $2.50(0.956)$ & $2.74(0.882)$ \\
\hline On target [\%] (SD) & $26.2 \%(0.029)$ & $32.6 \%(0.041)$ & $18.0 \%(0.103)$ \\
\hline $\operatorname{LLT}[\mathrm{n}](\%)$ & 119 (52.0\%) & $78(65.5 \%)$ & $41(34.5 \%)$ \\
\hline Statin [n] (\%) & 116 (97.5\%) & 76 (65.5\%) & 40 (34.5\%) \\
\hline Ezetimibe [n] (\%) & $5(4.2 \%)$ & $4(80.0 \%)$ & $1(20.0 \%)$ \\
\hline PCSK9 | [n] (\%) & $0(0.0 \%)$ & $0(0.0 \%)$ & $0(0.0 \%)$ \\
\hline
\end{tabular}

TABLE 1

Baseline information displayed as total and divided on to sex Legends:

IHD: Ischaemic heart disease; CVASC: Cerebral vascular disease; PAD: Peripheral artery disease; LDL-C: Low density lipoprotein; LLT: Lipid lowering therapy; Maximum: Highest measurement; Latest: Measurement at latest follow up visit

\section{TABLE 2}

Changes in LDL-C with or without therapy. A p-value $<0.05$ is considered significant Legends:

LDL-C: Low density lipoprotein; Maximum: Highest measurement; Latest: Measurement at latest follow up visit

\begin{tabular}{|c|c|c|c|c|c|c|}
\hline \multirow[t]{2}{*}{ TABLE 2} & & & & & \multicolumn{2}{|c|}{ Changes to LDL-C with or without therapy } \\
\hline & & $\mathrm{N}$ & $\begin{array}{l}\text { LDL-C, maximum } \\
{[\mathrm{mmol} / \mathrm{L}](\mathrm{SD})}\end{array}$ & $\begin{array}{l}\text { LDL-C, latest } \\
{[\mathrm{mmol} / \mathrm{L}](\mathrm{SD})}\end{array}$ & $\begin{array}{c}\text { Difference } \\
{[\mathrm{mmol} / /] \text { (SD) }}\end{array}$ & $\begin{array}{c}\text { Latest LDL-C }<1.8 \\
\mathrm{mmol} / \mathrm{L} \\
\text { Ratio }[\%](\mathrm{Cl})\end{array}$ \\
\hline \multirow{3}{*}{ Lipid lowering therapy } & Yes & 119 & $3.99(1.325)$ & $2.28(0.913)$ & $-1.72(1.234)$ & $38.7 \%(29.9-47.4)$ \\
\hline & No & 110 & $3.59(1.031)$ & $2.96(0.815)$ & $-0.63(0.882)$ & $12.7 \%(6.5-19.0)$ \\
\hline & p-value & --- & 0.011 & $<0.0001$ & $<0.0001$ & $<0.0001$ \\
\hline \multirow{3}{*}{ Statins } & Yes & 116 & $3.95(1.299)$ & $2.27(0.920)$ & $-1.68(1.224)$ & $39.7 \%(30.8-48.6)$ \\
\hline & No & 113 & $3.65(1.089)$ & $2.95(0.810)$ & $-0.70(0.966)$ & $12.4(6.3-18.5)$ \\
\hline & p-value & --- & 0.059 & $<0.0001$ & $<0.0001$ & $<0.0001$ \\
\hline
\end{tabular}

